\title{
BENACHTEILIGUNG IN DEN SCHULEN
}

Die erfolgreiche Integration von Schülerinnen und Schülern mit Migrationshintergrund ist in vielen OECD-Ländern ein wichtiges Politikziel. Der Erfolg eines Landes bei der Integration zugewanderter Schülerinnen und Schüler ist eine wichtige Messgröße der Qualität und Chancengerechtigkeit seines Bildungssystems und gibt darüber hinaus Auskunft über die Wirksamkeit seiner sozialpolitischen Maßnahmen im Allgemeinen. Die Unterschiede beim - selbst nach Berücksichtigung des sozioökonomischen Hintergrunds vorhandenen - Leistungsabstand zwischen Schülerinnen und Schülern mit und ohne Migrationshintergrund zwischen den Ländern lassen darauf schließen, dass der Politik bei der Beseitigung solcher Leistungsdifferenzen eine wichtige Rolle zukommt.

\section{Definition}

In PISA wird im Hinblick auf den Migrationsstatus zwischen drei Schülerkategorien unterschieden: a) Schülerinnen und Schüler ohne Migrationshintergrund, auch als einheimische Schüler

\section{Überblick}

Drei verschiedene Konzentrationsmessgrößen lassen sich untersuchen, um herauszufinden, welche sich auf die Schülerleistungen im Allgemeinen und die Leistungen zugewanderter Schülerinnen und Schüler im Besonderen auswirkt. Diese Messgrößen sind die verschiedenen Anteile an Schülerinnen und Schülern in den Schulen, die a) zugewandert sind, b) zugewandert sind und zu Hause eine andere Sprache sprechen, und c) unabhängig von ihrer Herkunft eine Mutter haben, die ein niedriges Bildungsniveau aufweist.

Bei den Leseleistungen der Schülerinnen und Schüler mit Migrationshintergrund ist der Prozentsatz der Schülerinnen und Schüler, deren Mütter ein niedriges Bildungsniveau aufweisen, in einer Schule in allen evaluierten Ländern (außer Estland) stärker negativ mit den individuellen Leistungen in Lesekompetenz korreliert als die beiden anderen Konzentrationsmessgrößen. In vielen europäischen Ländern ist der Zusammenhang zwischen den Leistungen zugewanderter Schülerinnen und Schüler sowie der Benachteiligung der Schulen besonders stark. Ausnahmen bilden die nordischen Länder, Irland und Spanien, auch wenn die Leistungen der Schülerinnen und Schüler mit Migrationshintergrund in diesen Ländern im Allgemeinen verglichen mit denen der einheimischen Schülerinnen und Schüler nicht immer günstig sind.

In den OECD-Ländern gibt es mehr zugewanderte als einheimische Schülerinnen und Schüler, deren Mütter ein niedriges Bildungsniveau aufweisen, an benachteiligten Schulen (56\% bzw. 50\%), außer in Irland, Israel, Italien, Neuseeland, Portugal und Spanien. Schülerinnen und Schüler mit Migrationshintergrund, deren Mütter ein hohes Bildungsniveau aufweisen, sind jedoch an benachteiligten Schulen stärker überrepräsentiert (im Durchschnitt 26\%) als einheimische Schülerinnen und Schüler (im Durchschnitt 14,5\%), außer in Brasilien, Estland, Israel und Norwegen. Das benachteiligte Quartil ist nicht durch Migrationsmerkmale gekennzeichnet, sondern vielmehr durch den Nachteil auf Grund des Bildungsstands der Mutter. bezeichnet, sind in dem Land geboren, in dem sie im Rahmen von PISA geprüft wurden, oder haben mindestens einen im Inland geborenen Elternteil, b) Schülerinnen und Schüler der zweiten Generation sind im Inland geboren, ihre Eltern sind aber im Ausland geboren, und c) Schülerinnen und Schüler der ersten Generation sind im Ausland geboren und haben im Ausland geborene Eltern. Bei diesem Indikator umfassen die Schülerinnen und Schüler mit Migrationshintergrund zugewanderte Schülerinnen und Schüler der ersten und zweiten Generation.

Als Schülerinnen und Schüler, deren Mütter ein niedriges Bildungsniveau aufweisen, werden Schüler bezeichnet, deren Mütter eine Schulbildung unter Sekundarstufe-II-Niveau haben. Schülerinnen und Schüler, deren Mütter einen Tertiärabschluss erworben haben, werden als Schülerinnen und Schüler, deren Mütter ein hohes Bildungsniveau aufweisen, bezeichnet.

Jede einbezogene Schule in den einzelnen Ländern wurde in ein Quartil eingestuft, das entsprechend dem geschätzten (gewichteten) Prozentsatz der Schülerinnen und Schüler der betreffenden Schule definiert wurde, deren Mütter ein niedriges Bildungsniveau aufweisen. Die benachteiligten Schulen entsprechen dem 4. Quartil, in dem der größte Anteil an Schülerinnen und Schülern zu finden ist, deren Mütter ein niedriges Bildungsniveau aufweisen. Die begünstigten Schulen sind dem 1. Quartil zugeordnet, wo der geringste Anteil an Schülerinnen und Schülern zu finden ist, deren Mütter ein niedriges Bildungsniveau aufweisen.

\section{Vergleichbarkeit}

PISA bezieht sich auf alle Schülerinnen und Schüler, die zum Zeitpunkt der Erhebung im Alter von 15 Jahren und 3 Monaten bis zu 16 Jahren und 2 Monaten sind und die mindestens sechs Jahre formaler Bildung abgeschlossen haben, gleichgültig in welcher Art von Bildungseinrichtung sie eingeschrieben sind und unabhängig davon, ob es sich um eine Ganztags- oder Halbtagsschule, eine allgemein- oder berufsbildende Einrichtung, eine öffentliche oder private Schule oder auch eine Auslandsschule in dem betreffenden Land handelt.

\section{Quelle}

- OECD (2012), Untapped Skills: Realising the Potential of Immigrant Students, PISA, OECD Publishing.

\section{Weitere Informationen}

\section{Analysen}

- OECD (2012), Equity and Quality in Education, Supporting Disadvantaged Students and Schools, OECD Publishing.

- OECD (2010), PISA 2009 Ergebnisse, OECD Publishing.

Statistiken

- OECD (2010), PISA 2009 at a Glance, OECD Publishing.

Zur Methodik

- OECD (2009), PISA 2009 Assessment Framework: Key Competencies in Reading, Mathematics and Science, PISA, OECD Publishing.

Online-Datenbanken

- OECD PISA Database.

\section{Websites}

- Internationale Schulleistungsstudie der OECD (PISA), www.pisa.oecd.org. 


\section{Schülerinnen und Schüler an benachteiligten Schulen und Durchschnittsergebnisse in Lesekompetenz nach dem Bildungsniveau der Mutter}

\begin{tabular}{|c|c|c|c|c|c|c|c|c|c|c|c|c|}
\hline & \multicolumn{4}{|c|}{$\begin{array}{l}\text { Schüler, deren Mütter ein niedriges Bildungsniveau aufweisen, } \\
\text { an benachteiligten Schulen, in Prozent aller Schüler, } \\
\text { deren Mütter ein niedriges Bildungsniveau aufweisen }\end{array}$} & \multicolumn{4}{|c|}{$\begin{array}{l}\text { Schüler, deren Mütter ein hohes Bildungsniveau aufweisen, } \\
\text { an benachteiligten Schulen, in Prozent aller Schüler, } \\
\text { deren Mütter ein hohes Bildungsniveau aufweisen }\end{array}$} & \multicolumn{4}{|c|}{ Mittlere Punktzahl auf der Gesamtskala Lesekompetenz } \\
\hline & \multicolumn{2}{|c|}{$\begin{array}{c}\text { Schüler mit } \\
\text { Migrationshintergrund }\end{array}$} & \multicolumn{2}{|c|}{$\begin{array}{l}\text { Schüler ohne } \\
\text { Migrationshintergrund }\end{array}$} & \multicolumn{2}{|c|}{$\begin{array}{l}\text { Schüler mit } \\
\text { Migrationshintergrund }\end{array}$} & \multicolumn{2}{|c|}{$\begin{array}{l}\text { Schüler ohne } \\
\text { Migrationshintergrund }\end{array}$} & \multicolumn{2}{|c|}{$\begin{array}{l}\text { Mütter weisen ein niedriges } \\
\text { Bildungsniveau auf }\end{array}$} & \multicolumn{2}{|c|}{$\begin{array}{l}\text { Mütter weisen ein hohes } \\
\text { Bildungsniveau auf }\end{array}$} \\
\hline & $\%$ & S.E. & $\%$ & S.E. & $\%$ & S.E. & $\%$ & S.E. & Mittelwert & S.E. & Mittelwert & S.E. \\
\hline Australien & 66.0 & 6.3 & 52.6 & 4.3 & 22.7 & 4.0 & 16.6 & 2.3 & 471 & 4.3 & 541 & 2.8 \\
\hline Belgien & 76.5 & 3.7 & 47.0 & 4.3 & 30.6 & 4.5 & 16.0 & 2.2 & 465 & 3.9 & 535 & 2.5 \\
\hline Chile & .. & .. & 46.7 & 5.2 & .. & .. & 7.3 & 1.4 & 416 & 3.4 & 487 & 3.6 \\
\hline Dänemark & 68.3 & 5.0 & 44.4 & 5.1 & 36.4 & 4.6 & 18.6 & 3.0 & 451 & 3.7 & 512 & 2.5 \\
\hline Deutschland & 58.5 & 6.4 & 45.8 & 4.9 & 31.3 & 5.3 & 13.0 & 2.5 & 448 & 4.2 & 529 & 4.2 \\
\hline Estland & .. & .. & 56.1 & 5.0 & 13.4 & 5.4 & 21.5 & 2.8 & 467 & 6.6 & 511 & 3.4 \\
\hline Finnland & .. & .. & 46.6 & 5.2 & 28.6 & 8.0 & 21.7 & 3.3 & 496 & 4.7 & 547 & 2.4 \\
\hline Frankreich & 62.8 & 6.4 & 43.8 & 5.3 & 36.5 & 7.4 & 13.1 & 2.4 & 456 & 4.6 & 529 & 4.4 \\
\hline Griechenland & 56.8 & 10.7 & 49.6 & 4.6 & 30.6 & 4.3 & 14.1 & 2.7 & 444 & 6.2 & 506 & 3.9 \\
\hline Irland & 26.2 & 8.8 & 47.2 & 5.6 & 28.6 & 6.0 & 15.6 & 3.2 & 461 & 4.0 & 519 & 3.3 \\
\hline Island & .. & .. & 43.2 & 1.9 & .. & .. & 17.6 & 0.8 & 477 & 3.2 & 520 & 2.2 \\
\hline Israel & 47.2 & 7.1 & 80.6 & 2.7 & 7.3 & 3.0 & 12.3 & 1.6 & 401 & 6.3 & 516 & 3.8 \\
\hline Italien & 40.1 & 5.4 & 43.7 & 2.1 & 18.7 & 4.2 & 11.0 & 1.0 & 459 & 2.6 & 503 & 2.4 \\
\hline Japan & .. & .. & 66.0 & 4.4 & .. &.. & 14.9 & 1.6 & 483 & 7.3 & 542 & 3.6 \\
\hline Kanada & 66.4 & 6.3 & 58.3 & 4.4 & 23.8 & 3.9 & 18.6 & 2.0 & 491 & 4.7 & 537 & 1.7 \\
\hline Korea & .. & .. & 54.7 & 5.0 & .. &.. & 14.7 & 2.8 & 504 & 7.2 & 555 & 4.9 \\
\hline Luxemburg & 48.7 & 1.8 & 27.4 & 2.2 & 15.7 & 1.8 & 11.1 & 0.9 & 436 & 2.6 & 503 & 2.7 \\
\hline Mexiko & 52.5 & 5.9 & 35.7 & 2.2 & 25.8 & 7.3 & 7.5 & 0.7 & 408 & 1.9 & 455 & 2.4 \\
\hline Neuseeland & 40.1 & 6.0 & 47.3 & 4.7 & 18.0 & 3.0 & 17.3 & 2.7 & 493 & 4.0 & 551 & 3.2 \\
\hline Niederlande & 71.4 & 6.0 & 40.6 & 5.2 & 41.7 & 9.1 & 15.3 & 2.7 & 479 & 5.8 & 526 & 5.5 \\
\hline Norwegen & 57.8 & 8.0 & 53.6 & 5.6 & 18.1 & 4.8 & 21.4 & 3.0 & 465 & 6.0 & 516 & 2.8 \\
\hline Österreich & 64.2 & 5.7 & 51.7 & 6.4 & 39.1 & 7.3 & 14.5 & 2.8 & 404 & 6.6 & 499 & 4.0 \\
\hline Polen & .. & .. & 55.3 & 5.3 & .. & .. & 14.9 & 3.1 & 444 & 5.1 & 553 & 3.9 \\
\hline Portugal & 12.4 & 3.8 & 37.0 & 4.2 & 10.8 & 3.3 & 6.7 & 1.2 & 470 & 3.2 & 531 & 4.5 \\
\hline Schweden & 59.7 & 8.1 & 45.0 & 5.1 & 37.5 & 6.7 & 19.6 & 2.8 & 447 & 6.1 & 513 & 3.2 \\
\hline Schweiz & 49.3 & 4.3 & 39.7 & 4.5 & 25.1 & 3.9 & 14.2 & 2.2 & 463 & 3.9 & 522 & 3.5 \\
\hline Slowak. Rep. & .. & .. & 83.1 & 3.7 & .. & .. & 18.4 & 3.0 & 384 & 11.3 & 503 & 4.2 \\
\hline Slowenien & 71.1 & 4.5 & 56.0 & 2.6 & 29.4 & 6.6 & 11.0 & 0.8 & 440 & 3.8 & 516 & 2.7 \\
\hline Spanien & 30.8 & 5.0 & 42.3 & 4.1 & 15.3 & 3.6 & 10.7 & 1.5 & 460 & 2.5 & 509 & 2.8 \\
\hline Tschech. Rep. & .. & .. & 72.2 & 4.7 & 25.5 & 9.5 & 21.6 & 3.5 & 432 & 7.4 & 496 & 4.9 \\
\hline Türkei &.. & .. & 30.0 & 3.8 & .. &.. & 2.7 & 0.9 & 454 & 3.2 & 523 & 7.5 \\
\hline Ungarn & .. & .. & 60.8 & 4.6 & .. & .. & 9.1 & 1.3 & 421 & 6.0 & 534 & 4.6 \\
\hline Ver. Königreich & 79.8 & 8.1 & 57.1 & 4.6 & 42.5 & 7.0 & 17.7 & 2.3 & 454 & 5.4 & 516 & 2.7 \\
\hline Ver. Staaten & 79.0 & 4.2 & 42.3 & 6.4 & 25.4 & 5.7 & 12.8 & 2.5 & 458 & 4.3 & 525 & 4.8 \\
\hline EU27 & .. & .. & .. &.. & .. &.. & .. & .. & .. & .. & .. & .. \\
\hline OECD & 55.9 & 1.3 & 50.1 & 0.8 & 26.1 & 1.1 & 14.5 & 0.4 & 453 & 0.9 & 520 & 0.6 \\
\hline Brasilien & 49.1 & 15.1 & 37.6 & 3.5 & 0.9 & 1.0 & 9.6 & 1.4 & 393 & 2.6 & 437 & 4.9 \\
\hline China & .. & .. & .. &.. &.. & .. &.. & .. &.. &.. &.. & .. \\
\hline Indien & .. & .. & .. & .. &.. &.. & .. & .. & .. & .. &.. & .. \\
\hline Indonesien &.. & .. & 35.5 & 4.6 & .. &.. & 4.6 & 1.4 & 390 & 3.2 & 437 & 8.3 \\
\hline Russ. Föderation &.. & .. & 75.9 & 6.1 & 23.0 & 5.6 & 21.9 & 3.6 & 397 & 12.3 & 468 & 3.2 \\
\hline Südafrika & .. &.. & .. &.. & .. &.. & .. &.. & .. &.. & .. & .. \\
\hline
\end{tabular}

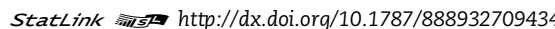

Korrelationen zwischen den Ergebnissen zugewanderter Schülerinnen und Schüler im Bereich Lesekompetenz und verschiedenen Messgrößen der Schülerkonzentration in den Schulen

Konzentration der Schüler, deren Mütter ein niedriges Bildungsniveau aufweisen

$\Delta$ Konzentration der Schüler mit Migrationshintergrund - Konzentration der Schüler mit Migrationshintergrund, die eine Fremdsprache sprechen

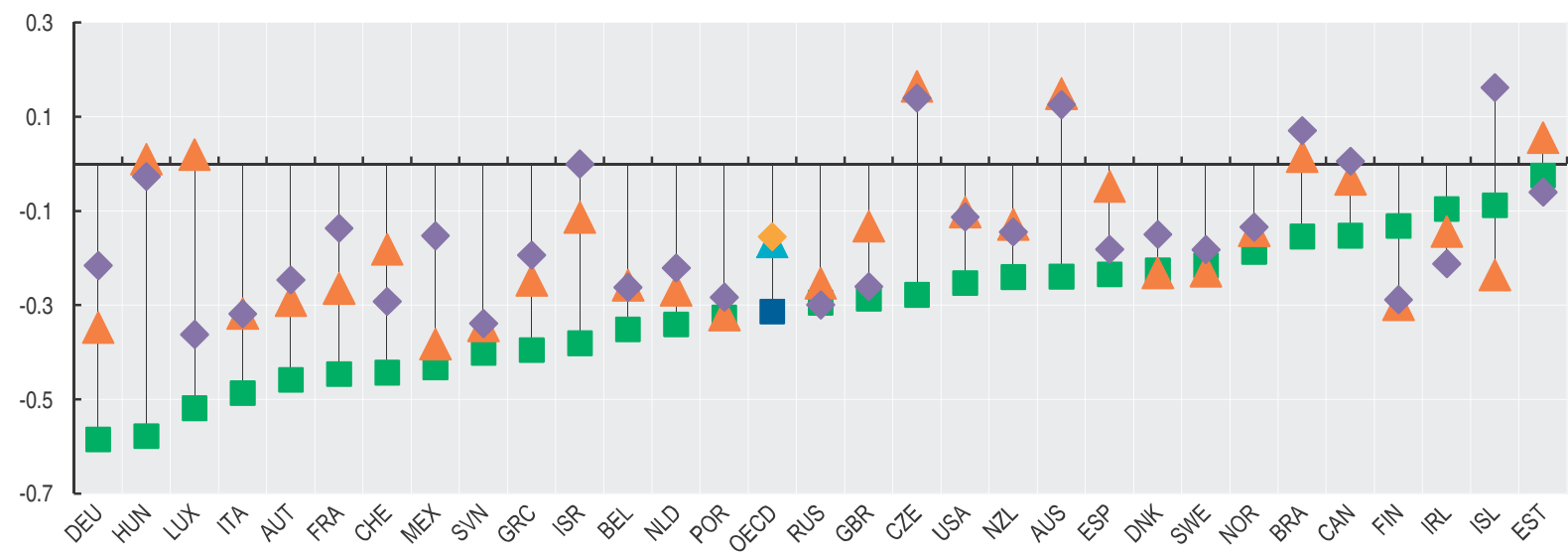




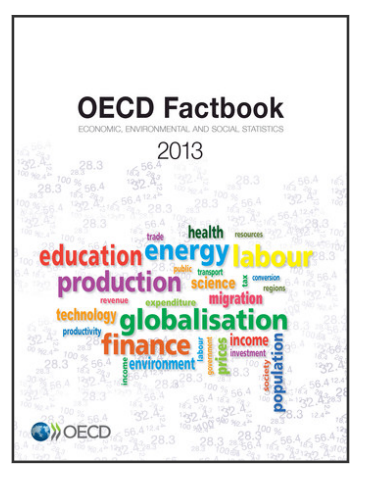

\section{From: \\ OECD Factbook 2013 \\ Economic, Environmental and Social Statistics}

Access the complete publication at:

https://doi.org/10.1787/factbook-2013-en

Please cite this chapter as:

OECD (2013), "Benachteiligung in den Schulen", in OECD Factbook 2013: Economic, Environmental and Social Statistics, OECD Publishing, Paris.

DOI: https://doi.org/10.1787/factbook-2013-73-de

Das vorliegende Dokument wird unter der Verantwortung des Generalsekretärs der OECD veröffentlicht. Die darin zum Ausdruck gebrachten Meinungen und Argumente spiegeln nicht zwangsläufig die offizielle Einstellung der OECD-

Mitgliedstaaten wider.

This document and any map included herein are without prejudice to the status of or sovereignty over any territory, to the delimitation of international frontiers and boundaries and to the name of any territory, city or area.

You can copy, download or print OECD content for your own use, and you can include excerpts from OECD publications, databases and multimedia products in your own documents, presentations, blogs, websites and teaching materials, provided that suitable acknowledgment of OECD as source and copyright owner is given. All requests for public or commercial use and translation rights should be submitted to rights@oecd.org. Requests for permission to photocopy portions of this material for public or commercial use shall be addressed directly to the Copyright Clearance Center (CCC) at info@copyright.com or the Centre français d'exploitation du droit de copie (CFC) at contact@cfcopies.com. 